\title{
Lesson of the month: Severe granulomatosis with polyangiitis (GPA): a diagnostic challenge during the COVID-19 pandemic
}

\author{
Authors: Qurratulain Qurratulain, ${ }^{A}$ Azeem Ahmed ${ }^{B}$ and Quentin Jones ${ }^{C}$
}

\begin{abstract}
We present the case of a 71-year-old woman with bilateral pneumonia who continued to deteriorate despite multiple courses of antibiotics. When dexamethasone was added to cover the possibility of COVID-19 pneumonia, she rapidly improved. Subsequently, she was found to have a strongly positive PR3 anti-nuclear cytoplasmic antibody (ANCA) and clinical features consistent with granulomatosis with polyangiitis (GPA) with upper respiratory tract and renal involvement. The case highlights how the COVID-19 pandemic can create new challenges in the diagnosis of GPA.
\end{abstract}

KEYWORDS: COVID-19, atypical pneumonia, granulomatosis with polyangiitis (GPA), anti-nuclear cytoplasmic antibodies (ANCA)

DOI: $10.7861 /$ clinmed.2020-0793

\section{Case presentation}

A 71-year-old woman with no past medical history presented to our acute medical unit with a 1-week history of non-productive cough, fever and shortness of breath. A chest X-ray (CXR; Fig 1) showed left mid-zone consolidation. Blood results revealed a markedly elevated C-reactive protein (CRP) of $260 \mathrm{mg} / \mathrm{L}$. Routine bloods including urea, creatinine and liver function tests were all normal. COVID-19 nasopharyngeal swabs were negative. Her CURB-65 score was 1. She was discharged home on oral antibiotics for 5 days with advice to self-isolate.

She re-presented 2 weeks later to the acute medical unit with fever, malaise, shortness of breath and cough with occasional mild haemoptysis. She had received two further courses of antibiotics in the community during the previous 2 weeks. Her oxygen saturations were $88 \%$ on air.

A repeat CXR revealed a worsening left-sided consolidation and new right upper lobe consolidation. Her CRP was 323 mg/L. A computed tomography (CT) of the chest (Fig 2) showed bilateral predominantly upper zone consolidation and small pleural effusions. She was treated with intravenous antibiotics for presumed severe pneumonia.

None of the initial tests to find the cause of the pneumonia were helpful. Her COVID-19 test was again negative. Blood, pleural fluid and sputum cultures were negative. Legionella and pneumococcal

Authors: ${ }^{\text {A }}$ respiratory senior house officer, Great Western Hospital, Swindon, UK; ${ }^{B}$ rheumatology consultant, Great Western

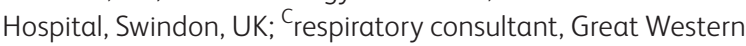
Hospital, Swindon, UK

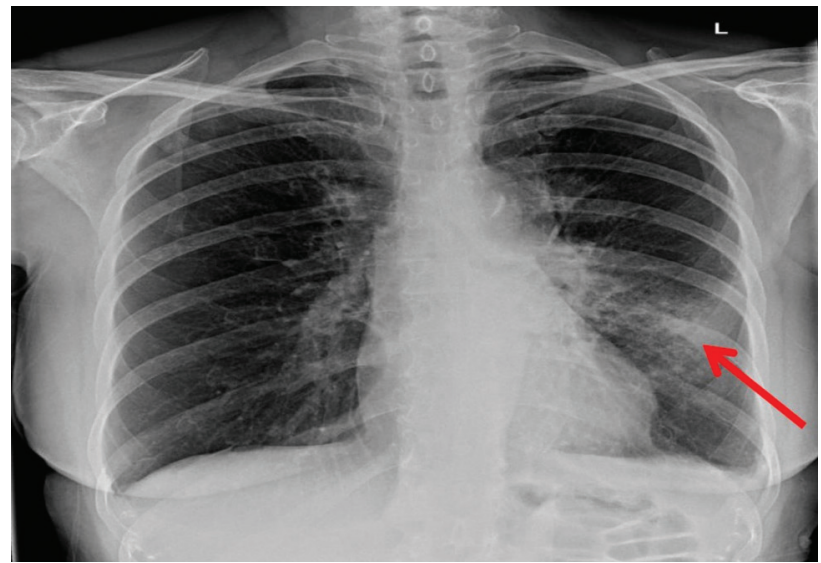

Fig 1. Initial chest X-ray showing left mid-zone consolidation (red arrow).

urinary antigen tests were negative, as were markers of fungal infection including aspergillus serology and B-D glucan. She also had a negative HIV test.

It was felt that COVID-19 pneumonia was still within the differential diagnosis despite negative testing. The patient was started on $6 \mathrm{mg}$ of dexamethasone daily orally as in the RECOVERY trial which had recently demonstrated benefit in hypoxic patients with COVID-19 pneumonia. ${ }^{1}$

After 24 hours, there was a marked clinical improvement and her inflammatory markers started to fall. She had a vasculitis screen sent earlier and the result now became available. Her anti-nuclear cytoplasmic antibodies (ANCA) test was positive with strongly positive PR3 antibody at $117 \mathrm{IU} / \mathrm{mL}$ (range 0-3.1).

A rheumatology review noted symptoms of rhinorrhoea, nasal crusting and epistaxis for the past few months, consistent with a diagnosis of granulomatosis with polyangiitis (GPA). A proteincreatinine ratio of $100 \mathrm{mg} / \mathrm{mmol}$ suggested renal involvement.

The patient was treated with oral prednisolone $1 \mathrm{mg} / \mathrm{kg}$ and started on rituximab infusion during her admission. Three days after receiving rituximab she was well enough to be discharged home.

\section{Discussion}

\section{Clinical features of GPA}

GPA is a life-threatening multisystem disease characterised by granulomatous inflammation, tissue necrosis and vasculitis in small and medium vessels. It can affect virtually any organ, but 


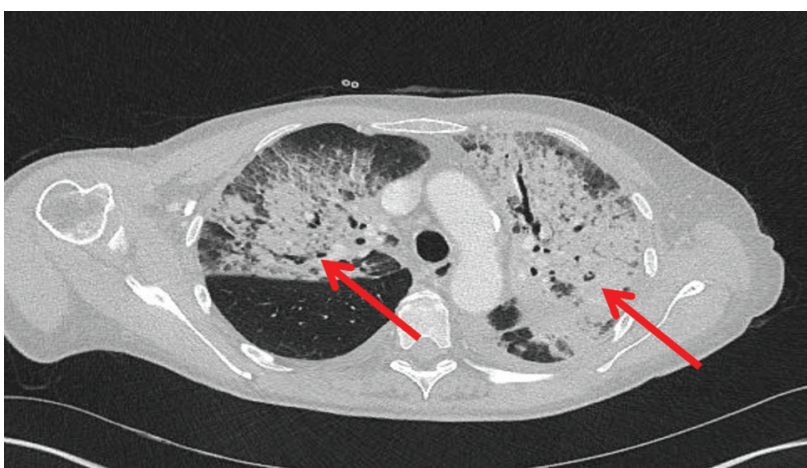

Fig 2. Computed tomography of the chest after 2 weeks showing development of extensive bilateral consolidation (red arrows) despite intravenous antibiotics.

most commonly affects the upper and lower airways and kidneys. Classic otorhinolaryngology symptoms may be the initial clinical manifestation of GPA because the upper respiratory tract is involved in $70-100 \%$ of cases. ${ }^{2}$ It can also present with limited involvement of a system. ${ }^{3}$

Pulmonary manifestations of the disease can occur in up to two-thirds of cases. The clinical picture can vary widely. Some patients are asymptomatic but have abnormal radiology. Others have symptoms similar to those of pneumonia and can present with fulminant pulmonary haemorrhage. Dyspnoea may arise from nasal disease, subglottal stenosis, endobronchial disease, parenchymal disease, pleural involvement or pulmonary artery involvement.

The CT findings in GPA are manifold and include areas of consolidation, mass like opacities, ground-glass shadowing, pleural effusions and cavitating nodules. Pulmonary haemorrhage can look like focal consolidation or sometimes diffuse perihilar consolidation.

\section{Diagnosing GPA}

Rapid diagnosis of GPA is important as early immunosuppression with pulsed cyclophosphamide or rituximab combined with glucocorticoids may be life- or organ-saving. ${ }^{4}$ However, there is often a delay in making the diagnosis and patients are frequently treated for pneumonia first.

A diagnosis of GPA should be considered in patients with atypical and non-resolving pneumonia. Taking a good history can pick up features suggestive of GPA with upper respiratory tract involvement, such as nasal crusting, nose bleeds, sinus symptoms and hearing loss. Haemoptysis, although non-specific, is a helpful clue. However, it is not necessarily present in GPA and is not always a prominent feature in the presentation, as in our patient.

The presence of renal failure or a significant amount of blood and protein in the urine should also raise the possibility of vasculitis. Urine should be dipped for blood and protein and a sample sent for microscopy and culture and protein/creatinine ratio.

Testing for ANCA is key in making the diagnosis. Eighty to ninety per cent of patients with GPA and lung involvement have c-ANCA antibodies to PR3. ${ }^{5}$ A tissue diagnosis should also be obtained whenever possible. The choice of biopsy site will depend on clinical features. Skin, nasal and renal biopsies are often helpful. When the lungs are involved, surgical lung biopsy has a high diagnostic yield, but the procedure is associated with morbidity. ${ }^{6}$

\section{Diagnosis of GPA during the COVID-19 pandemic}

The COVID-19 pandemic presents an added difficulty in making the diagnosis of GPA. The problem is that the clinical and radiological features can be quite similar and some commonly used diagnostic tests for COVID-19 have low sensitivity (nasal swabs about $63 \%$ and pharyngeal swabs $32 \%$ ). ${ }^{7}$ An observational study of patients with mild or moderate COVID-19 found symptoms similar to those seen in GPA, including loss of smell (70.2\%), nasal obstruction $(67.8 \%)$, cough $(63.2 \%)$, asthenia $(63.3 \%)$, myalgia $(62.5 \%)$, rhinorrhoea $(60.1 \%)$, gustatory dysfunction $(54.2 \%)$, sore throat $(52.9 \%)$ and fever $(45.4 \%){ }^{8}$ Haemoptysis is not always present in GPA and may occur in COVID-19 pneumonia. The clinical course of COVID-19 pneumonia and GPA can be similar with deterioration and protracted course. The case presented shows that patients with unrecognised GPA treated as COVID-19 pneumonia can improve after steroid administration, providing false reassurance and masking the real diagnosis.

\section{Conclusion}

This case highlights how a diagnosis of GPA should be considered in patients with atypical/non-resolving pneumonia, and how steroid treatment to cover the possibility of COVID-19 pneumonia can mask GPA. Revisiting the history and requesting further investigations, including ANCA, can be key in making the right diagnosis.

\section{References}

1 Horby P, Lim WS, Emberson JR et al. Dexamethasone in hospitalized patients with Covid-19 - preliminary report. New Engl J Med 2020;NEJMoa2021436 [Epub ahead of print].

2 Hartl DM, Aidan P, Brugiere $O$ et al. Wegener's granulomatosis presenting as a recurrence of chronic otitis media. Am J Otolaryngol 1998;19:54-60.

3 Cassan SM, Coles DT, Harrison EG. The concept of limited forms of Wegener's granulomatosis. Am J Med 1970;49:366-79.

4 Ntatsaki E, Carruthers D, Chakravarty K et al. BSR and BHPR guideline for the management of adults with ANCA-associated vasculitis. Rheumatology 2014;53:2306-9.

5 Schönermarck U, Lamprecht P, Csernok E, Gross WL. Prevalence and spectrum of rheumatic diseases associated with proteinase 3-antineutrophil cytoplasmic antibodies (ANCA) and myeloperoxidase-ANCA. Rheumatology 2001;40:178-84.

6 Travis WD, Hoffman GS, Leavitt RY et al. Surgical pathology of the lung in Wegener's granulomatosis. Review of 87 open lung biopsies from 67 patients. Am J Surg Pathol 1991;15:315-33.

7 Wang W, Xu Y, Gao R et al. Detection of SARS-CoV-2 in different types of clinical specimens. JAMA 2020;323:1843-4.

8 Lechien JR, Chiesa-Estomba CM, Place S et al. Clinical and epidemiological characteristics of 1,420 European patients with mild-tomoderate coronavirus disease 2019. J Intern Med 2020;288:335-44.

Address for correspondence: Dr Qurratulain Qurratulain, Respiratory Department, Great Western Hospital, Marlborough Road, Swindon SN3 6BB, UK.

Email: q.qurratulain@nhs.net 